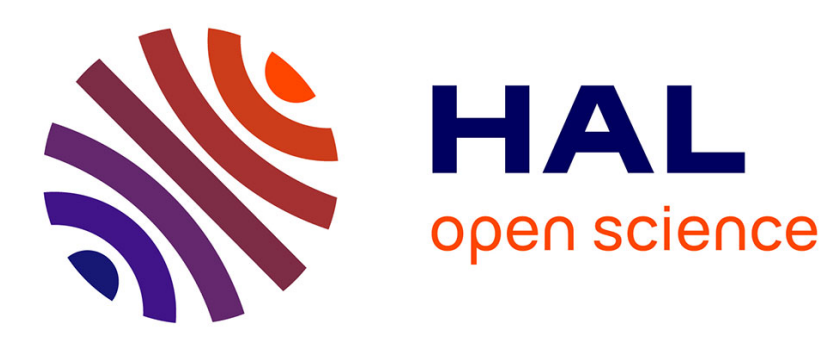

\title{
Patient Recollection of ICU Procedural Pain and Post ICU Burden
}

\author{
Kathleen Puntillo, Adeline Max, Marine Chaize, Gerald Chanques, Elie
}

Azoulay

\section{> To cite this version:}

Kathleen Puntillo, Adeline Max, Marine Chaize, Gerald Chanques, Elie Azoulay. Patient Recollection of ICU Procedural Pain and Post ICU Burden. Critical Care Medicine, 2016, 44 (11), pp.1988 - 1995. 10.1097/CCM.0000000000001875 . hal-01818500

\section{HAL Id: hal-01818500 \\ https://hal.science/hal-01818500}

Submitted on 16 Dec 2019

HAL is a multi-disciplinary open access archive for the deposit and dissemination of scientific research documents, whether they are published or not. The documents may come from teaching and research institutions in France or abroad, or from public or private research centers.
L'archive ouverte pluridisciplinaire HAL, est destinée au dépôt et à la diffusion de documents scientifiques de niveau recherche, publiés ou non, émanant des établissements d'enseignement et de recherche français ou étrangers, des laboratoires publics ou privés. 


\title{
Patient Recollection of ICU Procedural Pain and Post ICU Burden: The Memory Study*
}

\author{
Kathleen A. Puntillo, RN, PhD, FAAN, FCCM ${ }^{1}$; Adeline Max, MD²; Marine Chaize, $\mathrm{MS}^{3}$; \\ Gerald Chanques, $\mathrm{MD}, \mathrm{PhD}^{4}$; Elie Azoulay, $\mathrm{MD}, \mathrm{PhD}^{5}$
}

Objective: To assess patients' recollections of in-ICU procedural pain and its impact on post-ICU burden.

Design: Prospective longitudinal study of patients who underwent ICU procedures.

Setting: Thirty-four ICUs in France and Belgium.

Patients: Two hundred thirty-six patients who had undergone ICU procedures.

Intervention: None.

Measurements and Main Results: Patients were interviewed 3-16 months after hospitalization about: 1) recall of procedural pain intensity and pain distress (on 0-10 numeric rating scale); 2) current pain; that is, having pain in the past week that was not present before hospitalization; and 3) presence of traumatic stress

'Department of Physiological Nursing, University of California, San Francisco School of Nursing, San Francisco, CA.

${ }^{2}$ Medical field Transports, Ourson Bleu, Fontenay-Sous-Bois, France.

${ }^{3}$ AP-HP, Saint-Louis Hospital, Paris, France.

${ }^{4}$ Department of Anesthesiology \& Critical Care Medecine Montpellier University Regional Hospital, Saint Eloi Hospital Montpellier, Montpellier, France.

${ }^{5}$ Medical Intensive Care Unit, AP-HP, Saint-Louis Hospital, Paris, France.

This study was conducted at AP-HP, Hospital St Louis, Paris, France and the University of California, San Francisco, CA.

Supplemental digital content is available for this article. Direct URL citations appear in the printed text and are provided in the HTML and PDF versions of this article on the journal's website (http://journals.Iww.com/ccmjournal).

Supported, in part, by a grant from the European Society of Intensive Care Medicine/European Critical Care Research Network Award (ECCRN Established Investigator Award, 2009) and by an academic grant from AP-HP, Hospital Saint-Louis, Paris, France and Société de Réanimation de Langue Française.

Dr. Puntillo received funding from the Society of Critical Care Medicine. Dr. Max received funding from SRLF and Faculté de Médecine Paris Diderot. Her institution received funding from the Fondation de France and SRLF. Dr. Azoulay disclosed that none of his colleagues or his hospital have received grants from Fisher and Paeyckle. He received funding from Gilead (board member). His institution received funding from Astellas, Cubist, Alexion and from a Pfizer grant on antifungals. The remaining authors have disclosed that they do not have any potential conflicts of interest.

For information regarding this article, E-mail: kathleen.puntillo@ucsf.edu
(Impact of Events Scale). For patients who could rate recalled procedural pain intensity $(n=56)$ and pain distress $(n=43)$, both were significantly higher than their median (interquartile range) in ICU procedural pain scores (pain intensity: 5 [4-7] vs 3 [2.5-5], $p<0.001$; pain distress: 5 [2-6] vs 2 [0-6], $p=0.003$, respectively.) Current pain was reported in $14 \%$ of patients. When comparing patients with and without current pain, patients with current pain recalled even greater ICU procedural pain intensity and pain distress scores than patients without current pain: pain intensity, $8(6-8)$ versus 5 (3.25-7); $p=0.002$ and pain distress, 7 (5-8) versus $4(2-6) ; p=0.01$, respectively. Patients with current pain also had significantly higher Impact of Events Scale scores than those without current pain (8.5 [3.5-24] vs $2[0-10]$; $p<0.001)$. Conclusion: Many patients remembered ICU, with far fewer able to rate procedure-associated pain. For those able to do so, recalled pain intensity and pain distress scores were significantly greater than reported in ICU. One in seven patients was having current pain, recalling even higher ICU procedural pain scores and greater traumatic stress when compared with patients without current pain. Studies are needed to assess the impact of ICU procedural pain on post-ICU pain recall, pain status over time, and the relationship between postdischarge pain status and post-ICU burden. (

Key Words: critical care; intensive care unit; memory; pain; procedural pain

$\mathrm{P}$ ain is a significant problem for patients while in ICUs $(1,2)$ and after discharge (3-6). ICU procedures are one important source of pain for ICU patients (7-9). As a type of acute pain, procedural pain threatens tissue integrity and can initiate a series of psychologic, physiologic, and inflammatory stress responses (10). Many studies have documented patients' general pain while in $\operatorname{ICU}(1,2,11)$. Furthermore, other studies have identified non-ICU patients' recollections of procedures, such as colonoscopy and lithotripsy, where patients were able to make retrospective judgments about their procedure-related pain $(12,13)$. However, little has been reported about ICU patients' postdischarge recollection of ICU procedural pain and pain status after ICU. Therefore, we conducted 
a follow-up survey of French-speaking ICU patients who had participated in a larger study of ICU procedural pain (14). Our study aims were the following: 1 ) to investigate patients' memories of ICU procedural pain and its degree of intensity and distress; 2) to compare recalled procedural pain intensity and pain distress scores to the scores reported by the same patients when undergoing procedures in ICU; 3 ) to document the prevalence and severity of "current pain"; that is, pain in the past (interview) week that they did not have before hospitalization; and 4) to compare patients with and without current pain regarding ICU memories of procedural pain and current traumatic stress.

\section{MATERIALS AND METHODS}

Previously, we conducted a multinational, multisite study of pain associated with common ICU procedures (Europain) (14). The study coordinating center was a hospital in Paris, France. Patients were included in that study if they were adults 18 years old or older, were stable, were not receiving neuromuscular blocking agents, and were not delirious on the procedure day. Delirium was assessed by clinician data collectors in ICUs by a delirium assessment method used in their ICU. Patients in Europain were enrolled when they were to undergo one (or maximally two) of the study procedures. (List of procedures in Europain are given in Table 1 [14].) Ethics committee approval for the study was obtained at the study coordinating center in Paris and at the home institution of the principal investigator (K.P.). Institutional Review Board approval that met local legislation criteria at each site, including patient consent requirements, was mandatory for study participation. For this follow-up study, patients in 34 French-Speaking ICUs were approached before ICU discharge for consent to a follow-up telephone interview.

For this as well as the larger Europain (Paris, France) study (14), pain was conceptualized as having two dimensions: pain intensity and pain distress (15). Pain intensity is the severity of a pain sensation. Pain distress is an affective, or psychologic, response that relates to the unpleasantness that accompanies the sensation of pain (15). Numeric Rating Scales (NRS) were used to score ratings of procedural pain intensity and pain distress, where 0 was no pain (intensity or distress) and 10 was worst possible pain (intensity or distress).

For the follow-up study, we developed an interview guide with questions related to patient memories of ICU (16). Several questions related to ICU procedural pain, and questions related to pain at home. Content validity of the interview guide was performed by five nursing experts in pain and/or critical care. After this validation process, the interview guide was translated from English to French by a bilingual professional and back-translated by another bilingual translator according to the Brislin model for instrument translation for cross-cultural research $(17,18)$. The French version used in this study was pilot-tested on a discharged ICU patient who had consented to be in the study. Final revisions to the interview guide were made after the pilot interview.

\section{TABLE 1. Study Procedures, Operational Definitions, and Numbers of Patients Undergoing Each Procedure}

\begin{tabular}{|c|c|c|c|}
\hline Procedure & Operational Definition & $\begin{array}{l}\text { Larger Study } \\
\text { (Europain) (14), } n \text { (\%) }\end{array}$ & $\begin{array}{l}\text { Current } \\
\text { Study, } n(\%)\end{array}$ \\
\hline Turning & Turning the patient from supine to prone or vice versa & $873(18)$ & $46(20)$ \\
\hline Positioning & Moving the patient up or down in the bed & $371(8)$ & $8(3)$ \\
\hline Mobilization & Getting out of the bed or chair & $526(11)$ & $23(10)$ \\
\hline Respiratory exercises & Coughing, deep breathing & $439(9)$ & $26(11)$ \\
\hline Peripheral blood draw & Insertion of a catheter into a vein to draw out blood & $328(7)$ & $32(14)$ \\
\hline Peripheral IV line insertion & $\begin{array}{l}\text { Insertion of a catheter into a vein to give medications } \\
\text { or fluids }\end{array}$ & $315(7)$ & $12(5)$ \\
\hline Arterial line insertion & $\begin{array}{l}\text { Insertion of a catheter into an artery to monitor arterial } \\
\text { pressure and draw blood }\end{array}$ & $199(4)$ & $9(4)$ \\
\hline Endotracheal suctioning & $\begin{array}{l}\text { Insertion of a suction catheter into an endotracheal tube } \\
\text { to aspirate secretions }\end{array}$ & $767(16)$ & $31(13)$ \\
\hline Tracheal suctioning & $\begin{array}{l}\text { Insertion of a suction catheter into a tracheostomy to } \\
\text { aspirate secretions }\end{array}$ & $302(6)$ & $2(1)$ \\
\hline Chest tube removal & $\begin{array}{l}\text { Removal of a tube from the mediastinum or pleural } \\
\text { space }\end{array}$ & $292(6)$ & $13(6)$ \\
\hline Wound drain removal & $\begin{array}{l}\text { Removal of a drain from a wound (e.g., drain inserted } \\
\text { during abdominal surgery) }\end{array}$ & $75(2)$ & $10(4)$ \\
\hline Wound care & Cleaning, dressing change & $301(6)$ & $24(10)$ \\
\hline
\end{tabular}


We also used the Impact of Events scale (IES), a 15-item scale that measures stress reactions after traumatic events, with scores ranging from 0 to 75 . The IES includes an "Intrusion" Subscale Score (range, 0-35) and an "Avoidance" Subscale Score (range, 0-40) (19). Psychometric analyses show the IES to be a valid and reliable scale (20) that performs well as a selfreport measure of degree of subjective psychologic stress after some traumatic event (21).

All telephone interviews were made by two trained researchers at the Paris study center and one at a Belgium hospital between 3 and 16 months after the patient's discharge. The interviewers were under the supervision of a research team member with extensive experience in telephone survey techniques (M.C.). We developed guidelines and a script to be used during the telephone interview. At the beginning, patients were asked questions that evaluated their memory of the hospitalization, the consent process in ICU, and their continued willingness to be in the study and be interviewed. There were also directives about evaluating the patient's willingness to continue in the interview over time and to cease the interview if the patient desired. Patients were told the specific procedure to which we were referring. If patients had undergone more than one procedure in the study (maximum two procedures per patient), they were given the name of the first procedure as the focus of interview questions related to time in ICU. We asked their recall of pain intensity and pain distress associated with the procedure and whether they were having current pain, defined as pain in the past (interview) week that they did not have before hospitalization. Patients also completed the IES to measure their current traumatic stress.

After data were entered into a software program, data from a random sample of 50 patients were compared with their paper survey forms. There were no major discrepancies between the two datasets, and the few minor errors were corrected in the database. International Business Machines Statistical Package for the Social Sciences (SPSS) for Windows Version 21 (Armonk, NY, 2011) was used for performance of statistical analyses. Numerical variables are expressed as medians and interquartile ranges, and categoric variables are expressed in frequencies. Differences between groups were analyzed using a chi-square test for qualitative variables and Mann-Whitney $U$ and Wilcoxon signed rank tests for numerical variables, with a $p$ value of less than 0.05 considered statistically significant.

\section{RESULTS}

A total of 236 patients participated in the telephone interviews (Fig. 1 and Table 2). The median age was 57 (46-67), and the median ICU length of stay was 7 days (4-14). Most were medical patients. Patients' median Richmond Agitation Sedation Scale score, a measure of the degree of agitation or sedation (22), immediately prior to the procedure was $0(0-0)$, indicating that patients were alert and calm at that time. The percentages of patients with histories of anxiety, depression, chronic pain, or alcohol excess were not statistically significant between the current pain and no current pain groups. However, the

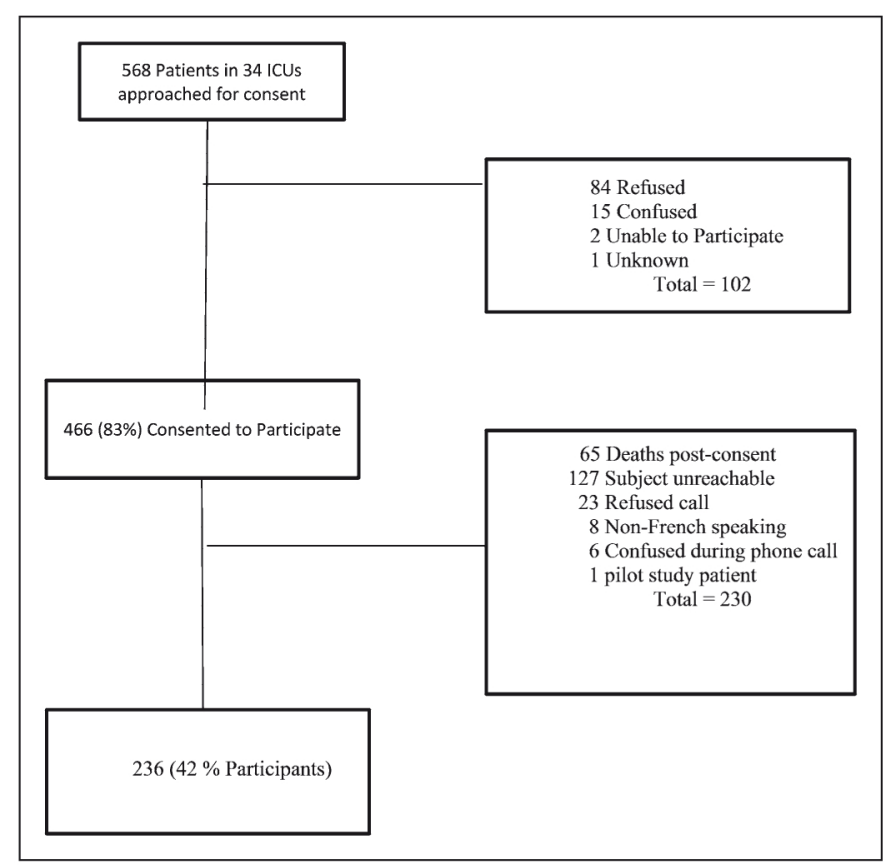

Figure 1. Patient recruitment flow diagram.

percentage of patients with chronic pain was substantially higher in the current pain group than in the no current pain group ( $n=5 ; 17 \%$ vs $n=12 ; 7 \%$, respectively.) The percentage of patients with chronic alcohol use was substantially lower in the current pain group than in the no current pain group ( $9 \%$ vs $20 \%)$. Five patients (17\%) with current pain had a prehospital history of opioid use, while nine patients (5\%) of patients without current pain had a prehospital history of opioid use, a statistically significant difference $(p=0.013)$.

\section{Procedural Pain Memories and in-ICU Procedural Pain Scores}

Most patients remembered being admitted to the hospital $(n=201 / 236 ; 85.5 \%)$ and being in the ICU $(n=181 / 236 ; 77 \%)$. Fewer patients remembered the procedure $(n=85 / 236 ; 36 \%)$. There were 61 patients who were able to specifically rate their recalled ICU procedural pain intensity (24\%) and 48 patients able to specifically rate their recalled procedural pain distress (18\%). We compared these recalled scores statistically to the scores provided by them when undergoing the procedure in ICU in patients with data at both times ( $n=56$, pain intensity; $n=43$, pain distress.) The recalled median NRS procedural pain intensity score was $5.0(4-7)$. This pain intensity score was significantly higher than the median NRS procedural pain intensity score of $3.0(2.5-5)$ reported by patients at the time of the ICU procedure $(p<0.001)$ (Table 3 ). The recalled median NRS procedural pain distress score was $5.0(2-6)$. This pain distress score was also significantly higher than the median NRS procedural pain distress score of $2.0(0-6)$ reported by patients at ICU-procedure time $(p=0.003)$ (Table 3$)$. Thus, patients recalled procedural pain intensity and distress scores to be greater than what they had reported in ICU (the degrees of recalled procedural pain intensity and distress according to 


\section{TABLE 2. Patient Demographic, Pre-hospital, and ICU Clinical Characteristics}

\begin{tabular}{|c|c|c|c|}
\hline Characteristics & $\begin{array}{l}\text { Total Group; } \boldsymbol{n}(\%) \\
\text { or Median (IQR) } \\
\quad(n=236)^{\mathrm{a}}\end{array}$ & $\begin{array}{c}\text { Patients With Current } \\
\text { Pain; } n(\%) \text { or Median (IQR) } \\
(n=32)^{\mathrm{a}}\end{array}$ & $\begin{array}{c}\text { Patients Without Current } \\
\text { Pain; } n(\%) \text { or Median (IQR) } \\
(n=200)^{\mathrm{a}}\end{array}$ \\
\hline Age (mean) & $57(46-67)$ & $57(33-64)$ & $57.5(47-68)$ \\
\hline Gender, male & $142(60)$ & $16(50)$ & $123(62)$ \\
\hline Primary diagnosis & $(n=231)$ & $(n=32)$ & $(n=195)$ \\
\hline Medical & $119(52)$ & $16(50)$ & $101(52)$ \\
\hline Surgical & $100(43)$ & $14(44)$ & $84(43)$ \\
\hline Trauma/burns & $12(5)$ & $2(6)$ & $10(5)$ \\
\hline $\begin{array}{l}\text { Sequential Organ Failure Assessment, } \\
\text { day of procedure }\end{array}$ & $3(1-5)$ & $3(1-5)$ & $3(1-5)$ \\
\hline $\begin{array}{l}\text { Richmond Agitation Sedation Scale, } \\
\text { day of procedure }\end{array}$ & $0(0-0)$ & $0(0-0)$ & $0(0-0)$ \\
\hline Mechanical ventilation in ICU, \% yes & $66(28)$ & $9(28)$ & $56(28)$ \\
\hline Days in ICU & $7(4-14)$ & $7(3-11)$ & $7(4-15)$ \\
\hline History of prehospital anxiety ${ }^{b}$ & $43 / 229(19)$ & $8 / 28(29)$ & $35 / 175(20)$ \\
\hline History of prehospital depression ${ }^{b}$ & $32 / 231(14)$ & $4 / 28(14)$ & $28 / 179(16)$ \\
\hline History of chronic pain ${ }^{b}$ & $18 / 230(8)$ & $5 / 30(17)$ & $12 / 180(7)$ \\
\hline History of chronic alcohol use ${ }^{b}$ & $42 / 232(18)$ & $3 / 32(9)$ & $39 / 191(20)$ \\
\hline History of prehospital opioid use ${ }^{b}$ & $14 / 216(7)$ & $5 / 29(17)^{\mathrm{c}}$ & $9 / 184(5)^{c}$ \\
\hline
\end{tabular}

$\mathrm{IQR}=$ interquartile range.

aAll numbers in columns do not add up to these totals due to some missing values.

ber hospital record review.

'Statistically significant difference in pre-hospital opioid use according to current pain status

each specific procedure are in Supplemental Table 1, Supplemental Digital Content 1, http://links.lww.com/CCM/B940).

\section{Differences in Procedural Pain Scores According to Time of Interview}

The range of time between the ICU procedure and home telephone interviews for the sub-sample of patient who were able to rate their recalled procedural pain intensity and distress was 9 months. Given this wide range, we performed a post hoc comparison of recalled procedural pain intensity and pain distress scores according to time from ICU procedure until time to interview: earlier or later. Patients who were interviewed earlier after ICU time $(<5$ mo; $n=29)$ recalled significantly lower procedural pain intensity than those who were interviewed later after ICU time ( $>5 \mathrm{mo} ; n=28$ ) (earlier NRS median $=5[3-6]$ vs later NRS median $=7[4-8]$; $p=0.007)$. In addition, there was a significant and positive correlation between number of days between discharge and the ICU interview and degree of procedural pain intensity $(r=0.39 ; p=0.003)$. Recalled procedural pain distress scores did not differ significantly according to interview time period (earlier NRS median $=4[2-5]$ vs later NRS median $=5[2-7]$;

\section{TABLE 3. Recalled and In-ICU Procedural Pain Intensity and Pain Distress Scores in Patients Per 0-10 Numeric Rating Scales}

Number of Patients Reporting - Pain Intensity and Distress
Median (IQR) Numeric Rating Scale Scores

p

Recalled procedural pain intensity $(n=56)$

$5.0(4-7)$

$<0.001$

Procedural pain intensity reported in ICU $(n=56)$

$3.0(2.5-5)$

Recalled procedural pain distress $(n=43)$

$5.0(2-6)$

$2.0(0-6)$

0.003 
$p=0.35)$. Nor was the correlation between number of days between discharge and the ICU interview and degree of procedural pain distress significant $(r=0.17 ; p=0.27$.

\section{Differences in Procedural Pain Scores: Medical Versus Surgical Patients}

Since our sample included both medical and surgical patients, a post hoc analysis was conducted to assess if results related to the memory of ICU procedural pain differed between those two groups. We compared recalled pain intensity scores from 28 medical and 23 surgical patients and recalled pain distress scores from 23 medical patients and 17 surgical patients. Recalled scores for both procedural pain intensity and procedural pain distress did not differ significantly between diagnostic groups.

\section{Traumatic Stress}

Regarding traumatic stress scores from 233 of 236 patients who completed the IES during the telephone interview, the median IES score was $3.0(0-11)$. Ten percent of the 233 patients $(n=23)$ had IES scores of 27 or greater (the cutoff score identified as predictive of PTSD) (23).

\section{Current Pain}

At the time of the follow-up telephone interview, $14 \%$ of 232 reporting patients $(n=32)$ reported having current pain during the week of the phone interview that they did not have before their ICU admission. Their median NRS pain intensity score at interview time $(n=32)$ was $5.5(4-7)$, and median pain distress score $(n=31)$ was $6.0(5-7)$. Their pain was getting better for $52 \%$, getting worse for $3 \%$, becoming unbearable for $7 \%$, and was staying the same for $39 \%$. For $58 \%$ $(n=19 / 32)$ of the patients with current pain, pain was keeping them from working; $62 \%(n=20 / 32)$ reported that their pain was restricting them from such life activities as dressing, walking, heavy lifting, home chores, and driving their car.

\section{Differences in Degrees of Recalled Procedural Pain and Current Traumatic Stress: Patients With Versus Without Current Pain}

Although the group was small, patients with current pain recalled notably higher ICU procedural pain scores when compared with patients without current pain. Of patients who provided scores, patients with current pain $(n=7)$ recalled significantly greater procedural pain intensity than did patients without current pain $(n=48)$ : median NRS equal to 8.0 (6-8) versus median NRS equal to 5.0 (3.25-7), $p$ value equal to 0.019 (Table 4). Patients with current pain $(n=7)$ also recalled significantly greater procedural pain distress than did patients without current pain $(n=35)$ (median NRS $=7.0(5-8)$ versus median NRS $=4.0(2-6) ; p=0.01)$. See Supplemental Table 2 (Supplemental Digital Content 1, http://links.lww.com/CCM/ B940) for frequency of types of procedures undergone by patients with and without current pain.

Regarding traumatic stress, 32 patients with current pain had a significantly higher median IES (stress) score (8.5 [3.5-24]) when compared with the 200 reporting patients without current pain $(2.0[0-10] ; p<0.001)$ (Table 4). Six $(19 \%)$ of the current pain patients had IES scores of greater than 27, a level at which there is a $75 \%$ chance of having PTSD. IES Intrusion and Avoidance scores also differed between patients with versus without current pain, with both scores being significantly higher in patients with current pain.

\section{DISCUSSION}

We conducted a follow-up investigation of discharged ICU patients who had participated in a procedural pain study months earlier, when they were patients in ICU. This study is unique in its attention to patient memories of their ICU procedural pain and how change with time occurred. Indeed,

\section{TABLE 4. Procedural Pain and Traumatic Stress Scores in Patients With and Without Current Pain Per 0-10 Numeric Rating Scales or Impact of Events Scale}

Pain Intensity, Pain Distress, and Impact of Events Scale Questions

Recalled procedural pain intensity (per NRS)

Procedural pain intensity reported in ICU (per NRS)

Recalled procedural pain distress (per NRS)

Procedural pain distress reported in ICU (per NRS)

Total IES score; range, 0-75

IES Intrusion Score; range, 0-35

IES Avoidance Score; range, 0-40

IES score with $75 \%$ chance of posttraumatic stress disorder (i.e., score $\geq 27$ )
Patients With Current Pain: $n$; Median (IQR) Scores

Patients Without Current Pain: $n$; Median (IQR)

p

$$
\begin{gathered}
7 ; 8.0(6-8) \\
7 ; 4.0(2-6) \\
7 ; 7.0(5-8) \\
7 ; 3.0(2-5) \\
n=32 \\
8.5(3.5-24)
\end{gathered}
$$

$$
\begin{gathered}
n=32 \\
5.5(0-12) \\
n=32 \\
3.7(0-11.5) \\
n=6 ; 19 \%
\end{gathered}
$$

$48 ; 5(3.25-7)$

0.019

48; $2.5(0-5)$

NS

$35 ; 4.0(2-6)$

0.01

35; $1.0(0-6)$

NS

$n=200$

$2(0-10)$

$n=200$

0 (0-5)

$n=200$

$0(0-5)$

$<0.001$

0.001

0.029

$n=17 ; 9 \%$

NS 
time may influence those memories and stress, especially for patients who were having pain at home that they did not have prior to their hospitalization. Certain observations can be drawn from our findings in relation to patient memory, pain memory overestimation, post-ICU pain status, and traumatic stress.

\section{Memory}

The vast majority of patients remembered being in ICU. However, many of our patients did not remember the procedure that they underwent or the degree of intensity and distress associated with their procedure. Chajut et al (24) noted that people may not remember all parts of events equally but may instead form an overall memory. Indeed, in many post-ICU studies, memories were variable concerning factual, delusional, and emotional memories (25-28); variability did not seem to be influenced by type of ICU sedation or sedation protocol $(25,28,29)$. Furthermore, while factual, delusional, and emotional questions asked of previous ICU patients were specific, our questions about rating the degree of procedural pain were even more specific and seemingly more difficult for some of our patients to answer.

\section{Pain Memory Overestimation}

Under every circumstance in which we asked about recalled procedural pain ratings and patients were able to provide a report, patients reported higher procedural pain scores than they had during ICU. While general memories of ICU may be retained, discordance may exist between actual events that occur in ICU and later reports by patients, and timing seems to be a major factor. Geng et al (30) describe a "watershed" time, between 3 and 7 weeks after an experience, when memory reflecting experiential knowledge may change to a more reconstructed memory. Thus, since our patients were interviewed no earlier than 3 months after ICU, their recall of greater procedural pain than reported in ICU may have been influenced by reconstructed memories of their ICU experiences. Indeed, our patients who did remember the degree of their procedural pain intensity and distress reported scores significantly higher than their in-ICU scores, with their median scores of 5 and 6 representing a moderate level of pain (31). Patients who were interviewed even later after ICU ( $>5 \mathrm{mo}$ ) reported having had significantly greater procedural pain intensity than patients interviewed less than 5 months after ICU. Thus, rather than the memory of procedural pain intensity decreasing over time, it increased in both situations.

Meaning of pain appears to influence retrospective pain reports. When comparing pain of childbirth per either vaginal or caesarean deliveries to postoperative pain from gynecological surgery, recalled degrees of pain intensity and unpleasantness were overestimated by the postoperative patients (13). In fact, vaginal delivery patients underestimated their earlier pain, and caesarean patients accurately estimated their earlier pain. The authors noted that surgery is "an unambiguously negative event" (pg 7), a description that most would agree is characteristic of time in an ICU. Thus, we suggest that type of pain, type of setting in which the pain is experienced, and temporal nature of the pain (e.g., time-limited such as during a caesarean procedure versus the waxing and waning of labor pain over several hours) may influence memories of ICU procedural pain.

Our patients' recalled procedural pain distress scores also reached the level of moderate pain. While pain intensity and distress scores are often highly correlated (32), they are distinct: pain intensity is the level of a pain sensation, while pain distress is an affective, or psychologic, response that relates to the unpleasantness that accompanies the sensation of pain (15). For clinicians, knowing their patient's level of pain distress could be just as important as knowing their patient's level of pain intensity since treatment decisions could differ. For example, while treatments were not addressed in the present study, ICU clinicians could consider analgesic agents prior to a procedure known to affect pain intensity and consider relaxation, information, or distraction to target procedural pain distress.

\section{Post-ICU Pain Status}

We found that $14 \%$, or approximately one out of seven, of our interviewed patients were having pain (i.e., current pain) at home in the past week that they did not have prior to their hospitalization. This percentage of people with postdischarge pain is lower than reported in other research studies, which ranged from $28 \%$ to $56 \%(3,6,33)$. Some patients in these studies may have had preexisting conditions that could have been the source of, or have influenced, their post-ICU pain. In our study, we specifically asked whether patients were having pain that was new since hospital admission; for 32 patients, this was the case. Of the patients with current pain, $17 \%$ (i.e., five of 29) had a history of opioid use prior to hospitalization. This percent was significantly larger than the 5\% (i.e., 9 of 184 patients) without current pain. While these numbers are small, it is important to consider prior use of opioids as a risk factor for pain after hospitalization.

The degrees of patients' current pain intensity and distress at interview time were moderate (31). Pain at these levels of severity could be a harbinger of persistent pain, a major disruption of the pain neurologic system (10). Processing of acute painful sensations in the brain can cause erratic firing of sensory nerves so that the system becomes short-circuited. As a result, sensory nerves that transmit painful stimuli can fire, even without any external stimuli (34). In other words, the experience of pain may become implanted in memory, in the somatosensory cortex that receives sensory nerve stimuli, causing hypersensitivity to previously nonpainful stimuli; pain can result and continue over time. Aggressive pain management at times when pain is anticipated remains important, especially if such management may decrease risks of neurologic changes that accompany persistent pain.

For seven of the patients with current pain, their rankings of recalled procedural pain intensity and distress were even higher than those reports of patients without current pain, with scores constituting severe pain (31). For them, memories 
of severe procedural pain may have been influenced by their current pain, a finding similar to other studies $(12,35)$.

\section{Traumatic Stress}

Our patients with current pain had significantly higher IES scores when compared with patients without current pain. It is very difficult to compare our results to other studies of PTSD in post-ICU patients due to heterogeneity of samples and PTSD measurement instruments (36), and we did not attempt to diagnose PTSD. However, in a recent meta-analysis of post-ICU organ transplant patients, the point prevalence of patients ( $n=1024)$ having substantial PTSD symptoms ranged from $0 \%$ to $46 \%$ (37). A recent meta-analysis by Parker et al (38) of 456 discharged general ICU patients found that $44 \%$ of their sample met criteria for having clinically important PTSD (i.e., a mean IES score of $>20$.) Our findings that $14 \%$ of our sample had median IES score of 8 , while not meeting the criterion of a powerful or severely stressful impact, is reflective of perceived stress at a level that could be a cause for clinical concern (39).

We acknowledge certain limitations to our study that suggest a cautious interpretation of findings. The sample in this analysis (as well as the sample in the original study) (14), although large, was one of convenience. However, our sample reported pain scores similar to the full Europain sample (14). The patient sample was also limited to French-speaking patients who were residents of France and Belgium. In addition, although patients were directed during the telephone interview to a specific procedure that they had undergone, their memory may have been an amalgamation of those and other experiences and may have been influenced by post-ICU events. Thus, we cannot isolate procedural pain as the only factor related their memories of ICU pain, their current pain, or emotional distress. While our sample was small, our patients without memory loss described their procedural pain recollections, their current pain, and their emotional status. Findings were statistically significant, novel, and important.

Other factors that could have influenced the patients' postICU memory and pain reports include preexisting PTSD, diagnosis, medications received, or ICU sedation and delirium. Although presence of delirium was an exclusion criterion, there were differences among ICUs in how clinicians determined delirium status. It may be that some patients in our study were delirious; however, previous studies noted no differences in ICU recall according to ICU delirium status (25) or sedation practices $(25,28,29)$. From our study, we do not know the impact of pain on the evolution of ICU patient delirium or the impact of delirium on recalled pain, topics for future research.

We cannot say with certainty that our 32 patients with current pain did, indeed, have neurologic abnormalities that accompany and define persistent pain (34). That is, we did not explore characteristics of their current pain, such as how many days per week they have had pain since hospitalization, whether their pain was constant, the sources of their pain, or neurologic characteristics of their pain. What we can say is that the perceptions of the 32 patients experiencing current pain were real for them, and these perceptions were associated with stress and life restrictions.

The relationship between pain overestimation, current pain, and current emotional distress is correlational, not causal, and some of the analyses were conducted on small subsamples. Future research on these relationships as a focus of attention may be warranted, given that undergoing painful procedures is an experience that most ICU patients share (7, 14). Our descriptive findings, as well as study limitations, could generate more specific research questions about pain after ICU discharge such as the following: 1) What are the characteristics of new pain postdischarge in patients reporting recall of ICU procedural pain, including its trajectory (number of days per months postdischarge), its neurologic characteristics (burning, stabbing, and sickening), and its timing (constant, transient, and brief); 2) What are risk factors for post-ICU pain intensity recall, post-ICU pain distress recall, and IES score, such as prehospital chronic pain, prehospital opioid use, procedure type (e.g., chest tube removal, wound drain removal, or endotracheal suctioning), preprocedural ICU pain, ICU postprocedure pain, ICU pain distress, and current pain among patients who can remember their ICU pain experience? Findings from this study could also inform future hypothesis-testing pain research regarding physical and psychologic stressors of ICU patients who experience procedural pain. One potential hypothesis could be: patients who experience moderateto-severe pain during an ICU procedure, such as chest tube removal, will recall pain at a higher intensity and have greater traumatic stress than patients who experience no or mild pain during an ICU procedure.

Until future relevant research findings are known, ICU clinicians can recognize that a subset of their patients may remember procedural pain for as long as 9 months after discharge, and this pain may be related to increased emotional stress. Preemptively treating procedural pain that is anticipated to be greater than mild in severity may positively influence the risk of post-ICU pain and emotional burden.

\section{CONCLUSIONS}

Approximately $20 \%$ of patients in our study provided recalled ratings of pain associated with a specific ICU procedure. These ratings were significantly higher than the levels of pain they originally reported while in ICU. In addition, almost one in seven of our patients was having pain on the interview day, months after ICU, that they did not have prior to hospitalization. Future qualitative research with patients who remember or do not remember their ICU pain may help identify nuances that can inform ICU pain care.

Current pain patients rated recalled ICU procedural pain as more severe, and their traumatic stress was higher than those who were not having current pain. Given that the ICU procedures we studied are a routine part of critical care, the unanticipated consequences of routine ICU care may be significant. That is, procedural pain experienced while in ICU could very well have substantial and continuous negative physical and psychologic ramifications for some patients. Future research 
is warranted that evaluates the results of treating ICU-related procedural pain to the maximum extent possible, with an eye to the possibility that acute pain that is not treated aggressively in ICU could be related to pain after ICU discharge. Such research could help in understanding how to better limit the development of post-ICU pain and, thus, positively affect ICU patients' memories and well-being.

\section{ACKNOWLEDGMENT}

We thank Pierre and Anne-Cécile Doucet, who conducted an independent audit of the database.

\section{REFERENCES}

1. Chanques $G$, Sebbane M, Barbotte $E$, et al: A prospective study of pain at rest: Incidence and characteristics of an unrecognized symptom in surgical and trauma versus medical intensive care unit patients. Anesthesiology 2007; 107:858-860

2. Gélinas C: Management of pain in cardiac surgery ICU patients: Have we improved over time? Intensive Crit Care Nurs 2007; 23:298-303

3. Lizana GF, Bota DP, De Cubber M, et al: Long-term outcome in ICU patients: What about quality of life? Intensive Care Med 2003; 29:1286-93

4. Lamer C, Harboun M, Knani L, et al: Quality of life after complicated elective surgery requiring intensive care. Intensive Care Med 2004; 30:1594-1601

5. Schelling G, Stoll C, Haller M, et al: Health-related quality of life and posttraumatic stress disorder in survivors of the acute respiratory distress syndrome. Crit Care Med 1998; 26:651-659

6. Battle CE, Lovett $\mathrm{S}$, Hutchings $\mathrm{H}$ : Chronic pain in survivors of critical illness: A retrospective analysis of incidence and risk factors. Crit Care 2013; 17:R101

7. Puntillo KA, White $C$, Morris $A B$, et al: Patients' perceptions and responses to procedural pain: Results from Thunder Project II. Am J Crit Care 2001; 10:238-251

8. Payen JF, Bru O, Bosson JL, et al: Assessing pain in critically ill sedated patients by using a behavioral pain scale. Crit Care Med $2001 ; 29: 2258-2263$

9. de Jong A, Molinari $N$, de Lattre $S$, et al: Decreasing severe pain and serious adverse events while moving intensive care unit patients: $A$ prospective interventional study (the NURSE-DO project). Crit Care 2013; 17:R74

10. Kyranou M, Puntillo K: The transition from acute to chronic pain: Might intensive care unit patients be at risk? Ann Intensive Care 2012; 2:36

11. Puntillo KA: Pain experiences of intensive care unit patients. Heart Lung 1990; 19:526-533

12. Redelmeier DA, Kahneman D: Patients' memories of painful medical treatments: Real-time and retrospective evaluations of two minimally invasive procedures. Pain 1996; 66:3-8

13. Babel P, Pieniazek L, Zarotynski D: The effect of the type of pain on the accuracy of memory of pain and affect. Eur J Pain 2014; 3:358-68

14. Puntillo KA, Max A, Timsit JF, et al: Determinants of procedural pain

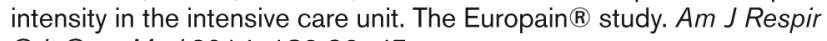
Crit Care Med 2014; 189:39-47

15. Price DD, Harkins SW, Baker C: Sensory-affective relationships among different types of clinical and experimental pain. Pain 1987; 28:297-307

16. Jones C, Humphris G, Griffiths RD: Preliminary validation of the ICUM tool for assessing memory of the intensive care experience. Clin Intensive Care 2000; 11:251-253
17. Jones PS, Lee JW, Phillips LR, et al: An adaptation of Brislin's translation model for cross-cultural research. Nurs Res 2001; 50:300-304

18. Brislin RW: Back-translation for cross-cultural research. J Cross-Cultural Psych 1970; 1:187-216

19. Horowitz M, Wilner N, Alvarez W: Impact of Event Scale: A measure of subjective stress. Psychosom Med 1979; 41:209-218

20. Sundin EC, Horowitz MJ: Impact of Event Scale: Psychometric properties. Br J Psychiatry 2002; 180:205-209

21. Sundin EC, Horowitz MJ: Horowitz's Impact of Event Scale evaluation of 20 years of use. Psychosom Med 2003; 65:870-876

22. Sessler CN, Gosnell MS, Grap MJ, et al: The Richmond AgitationSedation Scale: Validity and reliability in adult intensive care unit patients. Am J Respir Crit Care Med 2002; 166:1338-1344

23. Coffey SF, Gudmundsdottir B, Beck JG, et al: Screening for PTSD in motor vehicle accident survivors using the PSS-SR and IES. J Trauma Stress 2006; 19:119-128

24. Chajut E, Caspi A, Chen $R$, et al: In pain thou shalt bring forth children: The peak-and-end rule in recall of labor pain. Psychol Sci 2014; 25:2266-2271

25. Ethier C, Burry L, Martinez-Motta C, et al; Canadian Critical Care Trials Group: Recall of intensive care unit stay in patients managed with a sedation protocol or a sedation protocol with daily sedative interruption: A pilot study. J Crit Care 2011; 26:127-132

26. Ringdal M, Johansson L, Lundberg $D$, et al: Delusional memories from the intensive care unit-experienced by patients with physical trauma. Intensive Crit Care Nurs 2006; 22:346-354

27. Clark J, Voss L, Barnard J, et al: Implicit memory formation in sedated ICU patients after cardiac surgery. $\mathrm{Br} J$ Anaesth 2003; 91:810-814

28. Burry L, Cook D, Herridge M, et al; SLEAP Investigators; Canadian Critical Care Trials Group: Recall of ICU Stay in Patients Managed With a Sedation Protocol or a Sedation Protocol With Daily Interruption. Crit Care Med 2015; 43:2180-2190

29. Capuzzo M, Pinamonti A, Cingolani E, et al: Analgesia, sedation, and memory of intensive care. J Crit Care 2001; 16:83-89

30. Geng X, Chen Z, Lam W, et al: Hedonic evaluation over short and long retention intervals: The mechanism of the peak-end rule. J Behav Decis Mak 2012; 26:225-236

31. Serlin RC, Mendoza TR, Nakamura Y, et al: When is cancer pain mild, moderate or severe? Grading pain severity by its interference with function. Pain 1995; 61:277-284

32. Kunz M, Mylius V, Schepelmann K, et al: On the relationship between self-report and facial expression of pain. J Pain 2004; 5:368-376

33. Korosec Jagodic H, Jagodic K, Podbregar M: Long-term outcome and quality of life of patients treated in surgical intensive care: A comparison between sepsis and trauma. Crit Care 2006; 10:R134

34. Flor $\mathrm{H}$ : Painful memories. Can we train chronic pain patients to 'forget' their pain? EMBO Rep 2002; 3:288-291

35. Smith WB, Safer MA: Effects of present pain level on recall of chronic pain and medication use. Pain 1993; 55:355-361

36. Griffiths J, Fortune G, Barber V, et al: The prevalence of post traumatic stress disorder in survivors of ICU treatment: A systematic review. Intensive Care Med 2007; 33:1506-1518

37. Davydow DS, Lease ED, Reyes JD: Posttraumatic stress disorder in organ transplant recipients: A systematic review. Gen Hosp Psychiatry 2015; 37:387-398

38. Parker AM, Sricharoenchai T, Raparla S, et al: Posttraumatic stress disorder in critical illness survivors: A metaanalysis. Crit Care Med 2015; 43:1121-1129

39. Reed S: Measuring the Emotional Impact of an Event, 2013. [cited 2014 November 4]. Available at: http://www.psychotherapy-center.com/Measuring_the_Impact_of_an_Event.html. Accessed June 17, 2016 